\title{
Numerical Simulations of Stochastic Differential Equations with Multiple Conserved Quantities by Conservative Methods
}

\author{
Zhenyu Wang, Qiang Ma and Xiaohua Ding* \\ Department of Mathematics, Harbin Institute of Technology at Weihai, \\ Weihai 264209, PR. China.
}

Received 8 March 2021; Accepted (in revised version) 9 July 2021.

\begin{abstract}
The deterministic discrete gradient method for stochastic differential equations is extended to equations with multiple conserved quantities. The equations with multiple conserved quantities in the Stratonovich sense are written in the skew-gradient form, which is used in the construction of the stochastic discrete gradient method. It is shown that the stochastic discrete gradient method has the mean-square convergence order one and preserves all conserved quantities. Besides, for a given skew-gradient form, the stochastic discrete gradient method is equivalent to the stochastic projection method. Numerical examples confirm the theoretical results and show the effectiveness of the method.
\end{abstract}

AMS subject classifications: 65M10, 78A48

Key words: Stochastic differential equation, multiple conserved quantity, discrete gradient, projection, mean-square convergence.

\section{Introduction}

Stochastic differential equations (SDEs) are an important tool for describing stochastic phenomena arising in physics, engineering, economics, chemistry, and biology $[7,12,17,18$, 20]. However, analytical solutions of such equations are rarely available. Therefore, there are various numerical methods developed for their solution - cf. [2, 3, 11, 13, 25, 28-30]. In the last decades, the numerical methods preserving the geometric invariants along the flows, such as symplectic and Lie group structures, and quantities showing that the exact solution evolves on a manifold of the dimension smaller than that of $\mathbb{R}^{n}$, have been widely studied $[5,8,9,27,32]$. Such numerical approaches are called the structure-preserving or geometric numerical integration (GNI) methods. They have a number of advantages and provide reliable numerical solutions. Therefore, GNI for SDEs with geometric attributes

${ }^{*}$ Corresponding author. Email addresses: mathwzy@outlook.com (Z. Wang), hitmaqiang@hit.edu.cn (Q. Ma), mathdxh@hit.edu.cn (X. Ding) 
attracted a considerable attention $[4,14,19,24,33]$. In this work, we are mainly interested in SDEs with the conserved energy and momentum.

Consider the following general autonomous SDEs:

$$
\mathbf{d} x(t)=\sum_{i=0}^{d} G^{i}(x(t)) \circ \mathbf{d} W^{i}(t), \quad x(0)=x_{0},
$$

where "o" refers to the Stratonovich integral, $W^{0}(t):=t$ and $W^{i}(t), i=1, \ldots, d$ are independent one-dimensional standard Wiener processes defined on a complete filtered probability space $\left(\Omega, \mathscr{F}, \mathbb{P},\left\{\mathscr{F}_{t}\right\}_{t \in[0, T]}\right)$ and satisfying the usual conditions. Besides, the initial value $x_{0}$ is a $\mathbb{R}^{n}$-valued random variable such that $E\left|x_{0}\right|^{2}<\infty,|\cdot|$ the Euclidean norm, and the functions $G^{i}: \mathbb{R}^{n} \rightarrow \mathbb{R}^{n}, i=0, \ldots, d$, satisfy the conditions under which (1.1) has a unique solution [20].

To simplify the presentation, throughout this paper we use the Einstein's summation convention - i.e. repeated indices in a single term mean the summation of such terms over all range of the indices. Thus the Eq. (1.1) can be written as

$$
\mathbf{d} x(t)=G^{i}(x(t)) \circ \mathbf{d} W^{i}(t) .
$$

Assume that the Morse functions $I^{m}: \mathbb{R}^{n} \rightarrow \mathbb{R}, m=1, \ldots, M$ are conserved quantities of (1.2), i.e.

$$
\left(G^{i}\right)^{\top} \nabla I^{j}=0, \quad i=0, \ldots, d, \quad j=1, \ldots, M,
$$

where $\nabla I^{j}$ denotes the gradient of $I^{j}$. This means that the exact solution evolves on the manifold

$$
\mathscr{M}_{x_{0}}=\left\{y \in \mathbb{R}^{n} \mid I^{m}(y)=I^{m}\left(x_{0}\right) \text { for } m=1, \ldots M\right\} .
$$

When constructing numerical methods for SDEs with multiple conserved quantities, it is natural to require the numerical solutions to preserve all multiple conserved quantities. We note that there are two different sets of numerical methods which preserve conserved quantities of SDEs - viz. the ones that use the terms $I^{m}$ explicitly and the ones that do not. The latter include symplectic methods automatically preserving the quadratic Hamiltonian energy [24] and the methods preserving the matrix Lie group structure, which are equivalent to the preservation of conserved quantity determined by the corresponding Lie group $[1,19]$. The former comprise stochastic averaged vector field methods [6], stochastic projection methods $[15,34]$ and stochastic discrete gradient methods $[10,15]$.

It is worth noting that most of the existing works are devoted to SDEs with single conserved quantity. On the other hand, to the best of our knowledge there are only a few researches concerning conservative numerical methods for SDEs with multiple conserved quantities. Thus Chen et al. [4] modified the stochastic averaging vector field (AVF) method to preserve multiple conserved quantities, Zhou et al. [34] introduced multiple Lagrange multipliers and directly applied a linear projection method to SDEs with multiple conserved quantities. In comparison to the case of a single conserved quantity, this can increase the computational costs. In this paper, we extend a stochastic gradient method for SDEs with single conserved quantity [10] to SDEs with multiple conserved quantities and show that 
the stochastic linear projection method with multiple Lagrange multipliers can also be written in the same form.

The rest of the paper is organised as follows. In Section 2, the stochastic discrete gradient method for SDEs with multiple conserved quantities is presented and the mean-square convergence of the stochastic discrete gradient method based on a symmetric discrete gradient is illustrated. It is also proved that the stochastic linear projection method with multiple Lagrange multipliers can be written in the form of the stochastic discrete gradient method in a skew-gradient form. The numerical examples presented in Section 3, demonstrate the effectiveness of the method.

\section{A Stochastic Discrete Gradient Method}

In this section, we work with the SDEs (1.2) with $M$ conserved quantities $I^{m}, m=$ $1, \ldots, M$. First, considering the special case $M=1$, we obtain the classical stochastic gradient method for SDEs with single conserved quantity [10].

Theorem 2.1. Under the assumption $|\nabla I| \neq 0$, there exist skew-symmetric matrices $S^{i}, i=$ $0, \ldots, d$ such that

$$
G^{i}=S^{i} \nabla I
$$

Proof. Since $I$ is conserved, we have

$$
0=\mathbf{d} I=\frac{\mathbf{d} I}{\mathbf{d} x} \cdot \mathbf{d} x=(\mathbf{d} x)^{\top} \nabla I=\left(G^{i}\right)^{\top} \nabla I \circ \mathbf{d} W^{i},
$$

which implies $\left(G^{i}\right)^{\top} \nabla I=0$. Let

$$
S^{i}=\frac{1}{|\nabla I|^{2}}\left(G^{i}(\nabla I)^{\top}-\nabla I\left(G^{i}\right)^{\top}\right)=\frac{1}{|\nabla I|^{2}} G^{i} \wedge \nabla I,
$$

where $\wedge$ is the wedge product

$$
(u \wedge v)_{i j}=u_{i} v_{j}-u_{j} v_{i}, \quad u, v \in \mathbb{R}^{n} .
$$

Observing that $G^{i}(\nabla I)^{\top} /|\nabla I|^{2}$ maps $\nabla I$ to $G^{i}$, we can easily show that (2.2) is a particular solution of (2.1).

Generally, the choice of the skew-gradient form is not unique, since the solutions to (2.1) can be written as the sum of a particular solution and the solution to the homogeneous equation $S^{i} \nabla I=0$. The following proposition shows the general form of the solution to the homogeneous equation.

Proposition 2.1. For a given non-zero vector $v \in \mathbb{R}^{n}$, the general solution to homogeneous equation $S^{i} v=0$ can be written as

$$
S_{j k}^{i}=T_{j k l}^{i} v_{l},
$$

where $T^{i}$ are skew-symmetric 3-tensors, $i=0, \ldots, d$. 
Proof. For any $S^{i}$, let

$$
T_{j k l}^{i}=\frac{1}{|v|^{2}}\left(S_{j k}^{i} v_{l}+S_{k l}^{i} v_{j}+S_{l j}^{i} v_{k}\right)
$$

Since $S^{i} v=0$, i.e. $S_{j k}^{i} v_{k}=0$, we have

$$
T_{j k l}^{i} v_{l}=\frac{1}{|v|^{2}}\left(S_{j k}^{i} v_{l} v_{l}+S_{k l}^{i} v_{j} v_{l}+S_{l j}^{i} v_{k} v_{l}\right)=\frac{1}{|v|^{2}} S_{j k}^{i}\left(v_{l}\right)^{2}=S_{j k}^{i} .
$$

Rewriting (1.2) in the equivalent skew-gradient form

$$
\mathbf{d} x(t)=S^{i}(x(t)) \nabla I(x(t)) \circ \mathbf{d} W^{i}(t),
$$

we can construct a stochastic discrete gradient method based on the discrete gradient.

Definition 2.1 (cf. McLachlan et al. [21]). For a differentiable function $I$, the function $\bar{\nabla} I$ is the discrete gradient of $I$ if it is continuous and satisfies the equations

$$
\begin{aligned}
& \bar{\nabla} I\left(x^{\prime}, x\right) \cdot\left(x^{\prime}-x\right)=I\left(x^{\prime}\right)-I(x), \\
& \bar{\nabla} I(x, x)=\nabla I(x),
\end{aligned}
$$

for any $x^{\prime}, x \in \mathbb{R}^{n}$.

For example, the function $\bar{\nabla} I=\left[\bar{\nabla} I_{1}, \cdots, \bar{\nabla} I_{n}\right]^{\top}$, where

$$
\begin{aligned}
\bar{\nabla} I_{k}= & \frac{1}{2}\left(\frac{I\left(x_{1}^{\prime}, \cdots, x_{k-1}^{\prime}, x_{k}^{\prime}, x_{k+1}, \cdots, x_{n}\right)-I\left(x_{1}^{\prime}, \cdots, x_{k-1}^{\prime}, x_{k}, x_{k+1}, \cdots, x_{n}\right)}{x_{k}^{\prime}-x_{k}}\right. \\
& \left.+\frac{I\left(x_{1}, \cdots, x_{k-1}, x_{k}, x_{k+1}^{\prime}, \cdots, x_{n}^{\prime}\right)-I\left(x_{1}, \cdots, x_{k-1}, x_{k}^{\prime}, x_{k+1}^{\prime}, \cdots, x_{n}^{\prime}\right)}{x_{k}-x_{k}^{\prime}}\right)
\end{aligned}
$$

is a symmetric discrete gradient [21].

Consider the discretisation of the time interval $[0, T]$ with the step size $h$, and let $t_{l}=l h$, $l=0, \ldots, T / h$. The stochastic discrete gradient one-step approximation $x^{l+1}$ of the exact solution at time $t_{l+1}$ started from $x^{l}=x\left(t_{l}\right)$ has the form

$$
x^{l+1}=x^{l}+S^{i}\left(\frac{x^{l+1}+x^{l}}{2}\right) \bar{\nabla} I\left(x^{l}, x^{l+1}\right) \Delta W^{i} .
$$

The Eqs. (2.4) represents the stochastic discrete gradient method for SDEs with single conserved quantity. Hong et al. [10] proved that the stochastic discrete gradient method based on the one-step approximation (2.4) has the mean-square convergence order 1 .

Remark 2.1. As we can see, the construction of the stochastic discrete gradient method depends on the approximation of $S^{i}$ and on the discrete gradient $\bar{\nabla} I\left(x^{\prime}, x\right)$. According to Theorems 2.1 and (2.1), the choice of $S^{i}$ is not unique, so that we can obtain better numerical integrators by adding appropriate homogeneous solutions. Besides, if the discrete gradient $\bar{\nabla} I\left(x^{\prime}, x\right)$ is symmetric, the corresponding numerical method is also symmetric [31]. 
Similar to the case $M=1$, we first show that SDEs with $M>1$ conserved quantities can be written in a skew-gradient form and show that the choice of skew-gradient form is not unique. Then, based on a given skew-gradient form and given discrete gradients of $I^{1}, \cdots, I^{M}$, we construct a stochastic discrete gradient method for (1.2) with $M$ conserved quantities and prove that the stochastic discrete gradient method has the mean-square convergence order 1.

Theorem 2.2. Let $D=\left[\nabla I^{1}, \cdots, \nabla I^{M}\right], B=D^{\top} D$, i.e. $B_{j k}=\nabla I^{j} \cdot \nabla I^{k}$. Assume that $\nabla I^{1}, \cdots, \nabla I^{M}$ are linearly independent, so that $B$ is non-singular. Then there are skewsymmetric $(M+1)$-tensors $S^{i}$ such that

$$
G_{j}^{i}=S_{j j_{1} \cdots j_{M}}^{i} \nabla I_{j_{1}}^{1} \cdots \nabla I_{j_{M}}^{M}
$$

Proof. Since $G^{i} \cdot \nabla I^{j}=0$ for all $j$, we have

$$
\left(G^{i} \wedge \nabla I^{1} \wedge \cdots \wedge \nabla I^{M}\right) \cdot\left(\nabla I^{1}, \cdots, \nabla I^{M}\right)=G^{i} \operatorname{det} B .
$$

The non-singularity of $B$ yields that

$$
S_{j j_{1} \cdots j_{M}}^{i}=\frac{1}{\operatorname{det} B}\left(G^{i} \wedge \nabla I^{1} \wedge \cdots \wedge \nabla I^{M}\right)
$$

is a particular solution to (2.5).

Consider now the homogeneous solutions of (2.5).

Proposition 2.2. For skew-symmetric $(M+1)$-tensors $S^{i}$ and linearly independent vectors $v^{1}, \cdots, v^{M} \in \mathbb{R}^{n}$, the general solution to homogeneous equation

$$
S_{j j_{1} \cdots j_{M}}^{i} v_{j_{1}}^{1} \cdots v_{j_{M}}^{M}=0
$$

can be written as

$$
S_{j j_{1} \cdots j_{M}}^{i}=C_{j j_{1} \cdots j_{M} k}^{i l} v_{k}^{l}, \quad l=1, \ldots, M,
$$

where $C^{i l}$ is a skew-symmetric $(M+2)$-tensor corresponding to $v^{l}$.

Proof. Notice that the skew-symmetric $(M+1)$-tensor $S^{i}$ has $\left(\begin{array}{c}n \\ M+1\end{array}\right)$ independent components. Using the coordinate system $v^{j}:=e_{j}, j=1, \ldots, M$, where $e_{m}, m=1, \ldots, M$ are the unit vectors, we obtain $S_{j 12 \cdots M}^{i}=0$. The the skew symmetry of $S^{i}$ implies $S_{j 12 \cdots M}^{i}=0$ for $j \leq M$. Therefore, the skew-symmetric $S^{i}$ has $\left(\begin{array}{c}n \\ M+1\end{array}\right)-(n-M)$ independent components left. In order to establish the general form of solutions, we exploit (2.7), thus obtaining

$$
S^{i} \cdot\left(v^{1}, v^{2}, \cdots, v^{M}\right)=C^{i l} \cdot\left(v^{l}, v^{1}, v^{2}, \cdots, v^{M}\right)=0,
$$

which has $M\left(\begin{array}{c}n \\ M+2\end{array}\right)$ independent components. For $n>M+2$, we have

$$
M\left(\begin{array}{c}
n \\
M+2
\end{array}\right)>\left(\begin{array}{c}
n \\
M+1
\end{array}\right)-(n-M) .
$$


This means that there are sufficient solutions available. Then, we have to determine the terms $C^{i l}$ in (2.7). Let us illustrate this procedure for $C^{i 1}$. Note that

$$
\tilde{S}^{i}:=S^{i} \cdot\left(v^{2}, \cdots, v^{M}\right)=C^{i 1}\left(v^{1}, v^{2}, \cdots, v^{M}\right),
$$

because $\tilde{S} \cdot v^{j}=0$ for all $j$. This implies

$$
\left(\tilde{S}^{i} \wedge v^{1} \wedge \cdots \wedge v^{M}\right) \cdot\left(v^{1}, v^{2}, \cdots, v^{M}\right)=\tilde{S}^{i} \operatorname{det} B,
$$

where $B$ is a non-singular matrix with the entries $B_{j k}=v^{j} \cdot v^{k}$. Thus,

$$
C^{i 1}\left(v^{1}, v^{2}, \cdots, v^{M}\right)=\tilde{S}^{i}=\frac{1}{\operatorname{det} B}\left(\tilde{S}^{i} \wedge v^{1} \wedge \cdots \wedge v^{M}\right) \cdot\left(v^{1}, v^{2}, \cdots, v^{M}\right),
$$

which means

$$
C^{i 1}=\frac{1}{\operatorname{det} B}\left(\tilde{S}^{i} \wedge v^{1} \wedge \cdots \wedge v^{M}\right)
$$

Remark 2.2. According to [21], the basis of skew-symmetric $(M+1)$-tensors satisfying (2.6) is composed of skew-symmetric $(M+1)$-tensors satisfying the condition $A^{i} \cdot v^{k}=0$ for some $k$. They can be written as $A^{i}=C^{i k} \cdot v^{k}$ with the skew-symmetric $(M+2)$-tensor $C^{i k}=\left(1 /\left|v^{k}\right|^{2}\right) A^{i} \wedge v^{k}$.

Writing (1.2) in the equivalent skew-gradient form

$$
\mathbf{d} x_{j}(t)=S_{j j_{1} \cdots j_{M}}^{i} \nabla I_{j_{1}}^{1} \cdots \nabla I_{j_{M}}^{M} \circ \mathbf{d} W^{i}(t), \quad j=1, \ldots, n
$$

with

$$
S^{i}=\frac{1}{\operatorname{det} B} G^{i} \wedge \nabla I^{1} \wedge \cdots \wedge \nabla I^{M},
$$

we can obtain a one-step approximation of the stochastic discrete gradient method for (1.2) with $M$ conserved quantities — viz.

$$
x_{j}^{l+1}=x_{j}^{l}+\tilde{S}_{j j_{1} \cdots j_{M}}^{i} \bar{\nabla} I_{j_{1}}^{1} \cdots \bar{\nabla} I_{j_{M}}^{M} \Delta W^{i}, \quad j=1, \ldots, n,
$$

where

$$
\tilde{S}^{i}=\frac{1}{\operatorname{det} \tilde{B}} \tilde{G}^{i} \wedge \tilde{\nabla} I^{1} \wedge \cdots \wedge \tilde{\nabla} I^{M}
$$

is an approximation to

$$
S^{i}=\frac{1}{\operatorname{det} B} G^{i} \wedge \nabla I^{1} \wedge \cdots \wedge \nabla I^{M},
$$

$\tilde{G}^{i}$ and $\tilde{\nabla} I^{m}$ are approximations to $G^{i}$ and $\nabla I^{m}$.

For convenience, we denote $x^{l+1}$ by $x^{\prime}$ and $x^{l}$ by $x$, so that the above one-step approximation takes the form

$$
x_{j}^{\prime}=x_{j}+\tilde{S}_{j j_{1} \cdots j_{M}}^{i} \bar{\nabla} I_{j_{1}}^{1} \cdots \bar{\nabla} I_{j_{M}}^{M} \Delta W^{i}, \quad j=1, \ldots, n .
$$


Usually, the increments of Wiener processes $\Delta W_{j}, j=1, \ldots, r$ are represented by $\sqrt{h} \xi_{j}, j=$ $1, \ldots, r$ with independent $\mathscr{N}(0,1)$-distributed random variables $\xi_{j}$. The unboundedness of the standard Gaussian random variable yields that the random variable $\sqrt{h} \xi_{j}$ should be replaced by $\sqrt{h} \zeta_{h, j}$, where

$$
\zeta_{h, j}= \begin{cases}\xi_{j}, & \left|\xi_{j}\right| \leq A_{h}, \\ A_{h}, & \xi_{j}>A_{h}, \\ -A_{h}, & \xi_{j}<-A_{h}\end{cases}
$$

with $A_{h}=\sqrt{2 k|\ln h|}$ and a positive integer $k$. It is known that $\mathbb{E}\left(\xi_{j}-\zeta_{h, j}\right)^{2} \leq h^{k}$ [24]. According to $[16,34]$, the truncated methods have the same mean-square convergence order. Thus from now on, we assume that the increments $\sqrt{h} \xi_{j}$ are replaced by the truncated forms $\sqrt{h} \zeta_{h, j}$ with a properly chosen integer $k$.

\section{Properties of Stochastic Discrete Gradient Method}

In this section, we consider the conservation property and the mean-square convergence of the stochastic discrete gradient method. In addition, we show that the stochastic linear projection method with $M$ Lagrange multipliers can also be written as a stochastic discrete gradient method with a given skew-gradient form.

Theorem 3.1. Assume that $G^{i}, i=0, \ldots, d$ and $\nabla I^{m}, m=1, \ldots, M$ have bounded second moments and uniformly bounded derivatives up to a required order, $\tilde{G}^{i}$ and $\tilde{\nabla} I^{m}$ are, respectively, the approximations of $G^{i}$ and $\nabla I^{m}$ using the mid-point rule. Then the stochastic discrete gradient method based on the one-step approximation (2.9) and on the symmetric discrete gradient (2.3) satisfies the conditions

1. $I^{m}\left(x^{\prime}\right)=I^{m}(x)$ for all $m=1, \ldots, M$.

2. The stochastic discrete gradient method (2.9) has mean-square convergence order 1.

Proof. By the definition of the discrete gradient, we have

$$
I^{m}\left(x^{\prime}\right)-I^{m}(x)=\bar{\nabla} I^{m} \cdot\left(x^{\prime}-x\right)=\tilde{S}^{i} \cdot\left(\bar{\nabla} I^{1}, \cdots, \bar{\nabla} I^{M}\right) \cdot \bar{\nabla} I^{m} \Delta W^{i} .
$$

According to the skew symmetry of $S^{i}$, we have $I^{m}\left(x^{\prime}\right)=I^{m}(x)$. For simplicity, we illustrate the mean-square convergence in the case $M=2$. If $M>2$, the proof is analogous but more lengthy. For $M=2$, we have

$$
\mathbf{d} x_{j}(t)=S_{j j_{1} j_{2}}^{i} \nabla I_{j_{1}}^{1} \nabla I_{j_{2}}^{2} \circ \mathbf{d} W^{i}(t), \quad j=1, \ldots, n,
$$

where

$$
S_{j j_{1} j_{2}}^{i}=\frac{1}{\operatorname{det} B}\left|\begin{array}{ccc}
G_{j}^{i} & \nabla I_{j}^{1} & \nabla I_{j}^{2} \\
G_{j_{1}}^{i} & \nabla I_{j_{1}}^{1} & \nabla I_{j_{1}}^{2} \\
G_{j_{2}}^{i} & \nabla I_{j_{2}}^{1} & \nabla I_{j_{2}}^{2}
\end{array}\right| .
$$


Our assumptions give that $S_{j k l}^{i}$ has uniformly bounded derivatives up to the required order. Let $\Delta:=x^{\prime}-x$. The Taylor expansion yields

$$
\begin{aligned}
\tilde{S}_{j j_{1} j_{2}}^{i} & =S_{j j_{1} j_{2}}^{i}\left(x+\frac{\Delta}{2}\right) \\
& =S_{j j_{1} j_{2}}^{i}(x)+\frac{1}{2} \partial_{k} S_{j j_{1} j_{2}}^{i}(x) \Delta_{k}+\frac{1}{8} \partial_{k_{1} k_{2}} S_{j j_{1} j_{2}}^{i}\left(x+\theta \frac{\Delta}{2}\right) \Delta_{k_{1}} \Delta_{k_{2}} .
\end{aligned}
$$

The $k$-th component of $\bar{\nabla} I^{m}$ has the form

$$
\begin{aligned}
\bar{\nabla} I_{k}^{m}= & \partial_{k} I(x)+\frac{1}{2} \partial_{k l} I(x) \Delta_{l}+\frac{1}{4} \sum_{j_{1}, j_{2} \neq k} \partial_{k j_{1} j_{2}} I(x+\theta \Delta) \Delta_{j_{1}} \Delta_{j_{2}} \\
& +\frac{1}{4} \sum_{j \neq k} \partial_{k k j} I(x+\theta \Delta) \Delta_{k} \Delta_{j}+\frac{1}{6} \partial_{k k k} I(x+\theta \Delta)\left(\Delta_{k}\right)^{2} .
\end{aligned}
$$

Substituting (3.2) and (3.3) into the stochastic discrete gradient method defined by (2.9) yields

$$
x_{j}^{\prime}=x_{j}+\tilde{S}_{j j_{1} j_{2}}^{i} \bar{\nabla} I_{j_{1}}^{1} \bar{\nabla} I_{j_{2}}^{2} \Delta W^{i} .
$$

Therefore,

$$
\begin{aligned}
\Delta_{j}= & x_{j}^{\prime}-x_{j}=S_{j j_{1} j_{2}}^{i}(x) \partial_{j_{1}} I^{1}(x) \partial_{j_{2}} I^{2}(x) \Delta W^{i} \\
+ & \frac{1}{2}\left(\partial_{k} S_{j j_{1} j_{2}}^{i}(x) \partial_{j_{1}} I^{1}(x) \partial_{j_{2}} I^{2}(x)+S_{j j_{1} j_{2}}^{i}(x) \partial_{j_{1} k} I^{1}(x) \partial_{j_{2}} I^{2}(x)\right. \\
& \left.\quad+S_{j j_{1} j_{2}}^{i}(x) \partial_{j_{1}} I^{1}(x) \partial_{j_{2} k} I^{2}(x)\right) \Delta_{k} \Delta W^{i} \\
+ & \frac{1}{4}\left(S_{j j_{1} j_{2}}^{i}(x) \partial_{j_{1} k_{1}} I^{1}(x) \partial_{j_{2} k_{2}} I^{2}(x)+\partial_{k_{1}} S_{j j_{1} j_{2}}^{i}(x) \partial_{j_{1}} I^{1}(x) \partial_{j_{2} k_{2}} I^{2}(x)\right. \\
& \left.\quad+\partial_{k_{1}} S_{j j_{1} j_{2}}^{i}(x) \partial_{j_{1} k_{2}} I^{1}(x) \partial_{j_{2}} I^{2}(x)\right) \Delta_{k_{1}} \Delta_{k_{2}} \Delta W^{i}+\mathscr{O}\left(\Delta^{3}\right) .
\end{aligned}
$$

Iteratively, we have

$$
\begin{aligned}
x_{j}^{\prime}= & x_{j}+S_{j j_{1} j_{2}}^{i}(x) \partial_{j_{1}} I^{1}(x) \partial_{j_{2}} I^{2}(x) \Delta W^{i} \\
+ & \frac{1}{2}\left(\partial_{k} S_{j j_{1} j_{2}}^{i_{1}}(x) \partial_{j_{1}} I^{1}(x) \partial_{j_{2}} I^{2}(x)+S_{j j_{1} j_{2}}^{i_{1}}(x) \partial_{j_{1} k} I^{1}(x) \partial_{j_{2}} I^{2}(x)\right. \\
& \left.\quad+S_{j j_{1} j_{2}}^{i_{1}}(x) \partial_{j_{1}} I^{1}(x) \partial_{j_{2} k} I^{2}(x)\right) \\
& \times\left(S_{k j_{3} j_{4}}^{i_{2}}(x) \partial_{j_{3}} I^{1}(x) \partial_{j_{4}} I^{2}(x)\right) \Delta W^{i_{1}} \Delta W^{i_{2}} \\
+ & \frac{1}{2}\left(\partial_{k_{1}} S_{j j_{1} j_{2}}^{i_{1}}(x) \partial_{j_{1}} I^{1}(x) \partial_{j_{2}} I^{2}(x)+S_{j j_{1} j_{2}}^{i_{1}}(x) \partial_{j_{1} k_{1}} I^{1}(x) \partial_{j_{2}} I^{2}(x)\right. \\
& \left.\quad+S_{j j_{1} j_{2}}^{i_{1}}(x) \partial_{j_{1}} I^{1}(x) \partial_{j_{2} k_{1}} I^{2}(x)\right) \\
\times & \left(\partial_{k_{2}} S_{k_{1} j_{3} j_{4}}^{i_{2}}(x) \partial_{j_{3}} I^{1}(x) \partial_{j_{4}} I^{2}(x)+S_{k_{1} j_{3} j_{4}}^{i_{2}}(x) \partial_{j_{3} k_{2}} I^{1}(x) \partial_{j_{4}} I^{2}(x)\right. \\
& \left.\quad+S_{k_{1} j_{3} j_{4}}^{i_{2}}(x) \partial_{j_{3}} I^{1}(x) \partial_{j_{4} k_{2}} I^{2}(x)\right)
\end{aligned}
$$




$$
\begin{aligned}
& \times\left(S_{k_{2} j_{5} j_{6}}^{i_{3}}(x) \partial_{j_{5}} I^{1}(x) \partial_{j_{6}} I^{2}(x)\right) \Delta W^{i_{1}} \Delta W^{i_{2}} \Delta W^{i_{3}} \\
& +\frac{1}{4}\left(S_{j j_{1} j_{2}}^{i_{1}}(x) \partial_{j_{1} k_{1}} I^{1}(x) \partial_{j_{2} k_{2}} I^{2}(x)+\partial_{k_{1}} S_{j j_{1} j_{2}}^{i_{1}}(x) \partial_{j_{1}} I^{1}(x) \partial_{j_{2} k_{2}} I^{2}(x)\right. \\
& \left.\quad \quad+\partial_{k_{1}} S_{j j_{1} j_{2}}^{i_{1}}(x) \partial_{j_{1} k_{2}} I^{1}(x) \partial_{j_{2}} I^{2}(x)\right) \\
& \times\left(S_{k_{1} j_{3} j_{4}}^{i_{2}}(x) \partial_{j_{3}} I^{1}(x) \partial_{j_{4}} I^{2}(x)\right)\left(S_{k_{2} j_{5} j_{6}}^{i_{3}}(x) \partial_{j_{5}} I^{1}(x) \partial_{j_{6}} I^{2}(x)\right) \\
& \times \Delta W^{i_{1}} \Delta W^{i_{2}} \Delta W^{i_{3}}+\mathscr{O}\left(\Delta W^{i_{1}} \Delta W^{i_{2}} \Delta W^{i_{3}} \Delta W^{i_{4}}\right) .
\end{aligned}
$$

Note that the Milstein method for (2.8) has the form

$$
\begin{aligned}
\bar{x}_{j}^{\prime}= & x_{j}+S_{j j_{1} j_{2}}^{i}(x) \partial_{j_{1}} I^{1}(x) \partial_{j_{2}} I^{2}(x) \Delta W^{i} \\
+ & \frac{1}{2}\left(\partial_{k} S_{j_{1} j_{2}}^{i_{1}}(x) \partial_{j_{1}} I^{1}(x) \partial_{j_{2}} I^{2}(x)+S_{j j_{1} j_{2}}^{i_{1}}(x) \partial_{j_{1} k} I^{1}(x) \partial_{j_{2}} I^{2}(x)\right. \\
& \left.\quad+S_{j j_{1} j_{2}}^{i_{1}}(x) \partial_{j_{1}} I^{1}(x) \partial_{j_{2} k} I^{2}(x)\right) \\
& \times\left(S_{k j_{3} j_{4}}^{i_{2}}(x) \partial_{j_{3}} I^{1}(x) \partial_{j_{4}} I^{2}(x)\right) \Delta W^{i_{1}} \Delta W^{i_{2}} .
\end{aligned}
$$

Thus,

$$
\begin{aligned}
x_{j}^{\prime}-\bar{x}_{j}^{\prime}= & \frac{1}{2}\left(\partial_{k_{1}} S_{j j_{1} j_{2}}^{i_{1}}(x) \partial_{j_{1}} I^{1}(x) \partial_{j_{2}} I^{2}(x)+S_{j j_{1} j_{2}}^{i_{1}}(x) \partial_{j_{1} k_{1}} I^{1}(x) \partial_{j_{2}} I^{2}(x)\right. \\
& \left.\quad+S_{j j_{1} j_{2}}^{i_{1}}(x) \partial_{j_{1}} I^{1}(x) \partial_{j_{2} k_{1}} I^{2}(x)\right) \\
& \times\left(\partial_{k_{2}} S_{k_{1} j_{3} j_{4}}^{i_{2}}(x) \partial_{j_{3}} I^{1}(x) \partial_{j_{4}} I^{2}(x)+S_{k_{1} j_{3} j_{4}}^{i_{2}}(x) \partial_{j_{3} k_{2}} I^{1}(x) \partial_{j_{4}} I^{2}(x)\right. \\
& \left.\quad+S_{k_{1} j_{3} j_{4}}^{i_{2}}(x) \partial_{j_{3}} I^{1}(x) \partial_{j_{4} k_{2}} I^{2}(x)\right) \\
& \times\left(S_{k_{2} j_{5} j_{6}}^{i_{3}}(x) \partial_{j_{5}} I^{1}(x) \partial_{j_{6}} I^{2}(x)\right) \Delta W^{i_{1}} \Delta W^{i_{2}} \Delta W^{i_{3}} \\
+ & \frac{1}{4}\left(S_{j j_{1} j_{2}}^{i_{1}}(x) \partial_{j_{1} k_{1}} I^{1}(x) \partial_{j_{2} k_{2}} I^{2}(x)+\partial_{k_{1}} S_{j j_{1} j_{2}}^{i_{1}}(x) \partial_{j_{1}} I^{1}(x) \partial_{j_{2} k_{2}} I^{2}(x)\right. \\
& \left.\quad+\partial_{k_{1}} S_{j j_{1} j_{2}}^{i_{1}}(x) \partial_{j_{1} k_{2}} I^{1}(x) \partial_{j_{2}} I^{2}(x)\right) \\
& \times\left(S_{k_{1} j_{3} j_{4}}^{i_{2}}(x) \partial_{j_{3}} I^{1}(x) \partial_{j_{4}} I^{2}(x)\right)\left(S_{k_{2} j_{5} j_{6}}^{i_{3}}(x) \partial_{j_{5}} I^{1}(x) \partial_{j_{6}} I^{2}(x)\right) \\
& \times \Delta W^{i_{1}} \Delta W^{i_{2}} \Delta W^{i_{3}}+\mathscr{O}\left(\Delta W^{i_{1}} \Delta W^{i_{2}} \Delta W^{i_{3}} \Delta W^{i_{4}}\right) .
\end{aligned}
$$

It follows that

$$
\left|\mathbb{E}\left(x_{j}^{\prime}-\bar{x}_{j}^{\prime}\right)\right|=\mathscr{O}\left(h^{2}\right), \quad\left(\mathbb{E}\left(x_{j}^{\prime}-\bar{x}_{j}^{\prime}\right)^{2}\right)^{1 / 2}=\mathscr{O}\left(h^{3 / 2}\right) .
$$

By [23, Theorem 1.1] and the triangle inequality, we have

$$
\left|\mathbb{E}\left(x_{j}^{\prime}-x_{j}\left(t^{l}\right)\right)\right|=\mathscr{O}\left(h^{2}\right), \quad\left(\mathbb{E}\left(x_{j}^{\prime}-x_{j}\left(t^{l}\right)\right)^{2}\right)^{1 / 2}=\mathscr{O}\left(h^{3 / 2}\right) .
$$

Thus the stochastic discrete gradient method (3.4) has the mean-square convergence of order 1. 
Now we show that the stochastic linear projection method with $M$ Lagrange multipliers can also be written as the stochastic discrete gradient method with a skew-gradient form.

Consider the one-step numerical methods for SDEs (1.2)

$$
x^{\prime}=x+\tilde{G}^{i}\left(x^{\prime}, x, h, \Delta W^{r}, r=1, \ldots, d\right) \Delta W^{i} .
$$

The following proposition provide the conditions for preserving all multiple conserved quantities by the numerical method (3.5).

Proposition 3.1. The numerical method (3.5) preserve all multiple conserved quantities if and only if

$$
\tilde{G}^{i}\left(x^{\prime}, x, h, \Delta W^{r}, r=1, \ldots, d\right) \in \operatorname{span}\left\{\bar{\nabla} I^{1}, \cdots, \bar{\nabla} I^{M}\right\}^{\perp} .
$$

Proof. Using the definition of the discrete gradient, we write

$$
I^{m}\left(x^{\prime}\right)-I^{m}(x)=\bar{\nabla} I^{m} \cdot\left(x^{\prime}-x\right)=\bar{\nabla} I^{m} \cdot \tilde{G}^{i} \Delta W^{i} .
$$

In order to preserve conserved quantities, we require that $I^{m}\left(x^{\prime}\right)-I^{m}(x)=0$, i.e. $\bar{\nabla} I^{m} \tilde{G}^{i}=$ 0 , which means $\tilde{G}^{i} \in \operatorname{span}\left\{\bar{\nabla} I^{1}, \cdots, \bar{\nabla} I^{M}\right\}^{\perp}$.

Recall that the main idea of the standard stochastic linear projection method [34] is to use the Lagrange multiplier $\lambda \in \mathbb{R}^{M}$ and to project the numerical solutions generated by a classical numerical method into the manifold

$$
\mathscr{M}_{x_{0}}=\left\{y \in \mathbb{R}^{n} \mid I^{m}(y)=I^{m}\left(x_{0}\right) \text { for all } m=1, \ldots M\right\},
$$

determined by the multiple conserved quantities of the original SDEs.

The following lemma shows that the standard stochastic linear projection method can be written as an equivalent stochastic projection method determined by the projection operator

$$
P:=\mathbb{I}-\tilde{D}\left(\bar{D}^{\top} \tilde{D}\right)^{-1} \bar{D}^{\top},
$$

where $\mathbb{I}$ is the identity operator, $\bar{D}=\left[\bar{\nabla} I^{1}, \cdots, \bar{\nabla} I^{M}\right]$ and $\tilde{D}=\left[\tilde{\nabla} I^{1}, \cdots, \tilde{\nabla} I^{M}\right]$.

Lemma 3.1. Let $\lambda$ be the Lagrange multiplier determined by $I^{m}\left(x^{\prime}\right)=I^{m}(x)$ for all $m$. If $\left(\bar{D}^{\top} \tilde{D}\right)$ is invertible, then the standard stochastic linear projection method

$$
x^{\prime}=x+\tilde{G}^{i}\left(x^{\prime}, x, h, \Delta W^{r}, r=1, \ldots, d\right) \Delta W^{i}+\tilde{D} \lambda
$$

is equivalent to the stochastic projection method determined by the projection operator $P$,

$$
x^{\prime}=x+P \tilde{G}^{i} \Delta W^{i} .
$$

Proof. For the standard stochastic linear projection method (3.6), we have

$$
0=I^{m}\left(x^{\prime}\right)-I^{m}(x)=\bar{\nabla} I^{m} \cdot\left(x^{\prime}-x\right)=\bar{\nabla} I^{m} \cdot\left(\tilde{G}^{i} \Delta W^{i}+\tilde{D} \lambda\right) .
$$

This means that

$$
0=\left(\bar{\nabla} I^{m}\right)^{\top} \tilde{G}^{i} \Delta W^{i}+\left(\bar{\nabla} I^{m}\right)^{\top} \tilde{D} \lambda
$$


Therefore,

$$
0=\bar{D}^{\top} \tilde{G}^{i} \Delta W^{i}+\bar{D}^{\top} \tilde{D} \lambda
$$

and, consequently,

$$
\lambda=-\left(\bar{D}^{\top} \tilde{D}\right)^{-1} \bar{D}^{\top} \tilde{G}^{i} \Delta W^{i} .
$$

Substituting $\lambda$ into (3.6) gives

$$
\begin{aligned}
x^{\prime} & =x+\tilde{G}^{i} \Delta W^{i}-\tilde{D}\left(\bar{D}^{\top} \tilde{D}\right)^{-1} \bar{D}^{\top} \tilde{G}^{i} \Delta W^{i} \\
& =x+\left(\mathbb{I}-\tilde{D}\left(\bar{D}^{\top} \tilde{D}\right)^{-1} \bar{D}^{\top}\right) \tilde{G}^{i} \Delta W^{i} \\
& =x+P \tilde{G}^{i} \Delta W^{i} .
\end{aligned}
$$

Let us illustrate the equivalence between the stochastic discrete gradient method (2.9) with

$$
\tilde{S}^{i}=\frac{1}{\operatorname{det}\left(\bar{D}^{\top} \tilde{D}\right)} \tilde{G}^{i} \wedge \tilde{\nabla} I^{1} \wedge \cdots \wedge \tilde{\nabla} I^{M}
$$

and the stochastic linear projection method (3.7). First, we recall two useful lemmas.

Lemma 3.2 (cf. Meyer [22]). For matrix $A \in \mathbb{R}^{n \times n}$ and vectors $c, d \in \mathbb{R}^{n}$, we have

$$
\operatorname{det}\left(A+c d^{\top}\right)=\operatorname{det}(A)\left(1+d^{\top} A^{-1} c\right) \text {. }
$$

Lemma 3.3 (cf. Meyer [22]). For vectors $u^{1}, \cdots, u^{M}, v^{1}, \cdots, v^{M} \in \mathbb{R}^{n}$ and matrices $U=$ $\left[u^{1}, \cdots, u^{M}\right] \in \mathbb{R}^{n \times M}, V=\left[v^{1}, \cdots, v^{M}\right] \in \mathbb{R}^{n \times M}$, we have

$$
\left(u^{1} \wedge \cdots \wedge u^{M}\right)_{j_{1} \cdots j_{M}} v_{j_{1}}^{1} \cdots v_{j_{M}}^{M}=\operatorname{det}\left(U^{\top} V\right)
$$

Now, we can present the equivalence theorem.

Theorem 3.2. Under the assumptions of Theorem 3.1 and (3.1), the stochastic discrete gradient method (2.9) with the skew-gradient form

$$
\tilde{S}^{i}=\frac{1}{\operatorname{det}\left(\bar{D}^{\top} \tilde{D}\right)} \tilde{G}^{i} \wedge \tilde{\nabla} I^{1} \wedge \cdots \wedge \tilde{\nabla} I^{M}
$$

is equivalent to the stochastic linear projection method (3.7).

Proof. For any vector $v \in \mathbb{R}^{n}$, we have

$$
\begin{aligned}
& \left(\tilde{G}^{i} \wedge \tilde{\nabla} I^{1} \wedge \cdots \wedge \tilde{\nabla} I^{M}\right)_{j j_{1} \cdots j_{M}} v_{j} \bar{\nabla} I_{j_{1}}^{1} \cdots \bar{\nabla} I_{j_{M}}^{M} \\
& =\operatorname{det}\left(\left[\tilde{G}^{i} \tilde{\nabla} I^{1} \cdots \tilde{\nabla} I^{M}\right]^{\top}\left[\nu \bar{\nabla} I^{1} \cdots \bar{\nabla} I^{M}\right]\right) \\
& =\operatorname{det}\left(\begin{array}{cccc}
\tilde{G}^{i} \cdot v & \tilde{G}^{i} \cdot \bar{\nabla} I^{1} & \ldots & \tilde{G}^{i} \cdot \bar{\nabla} I^{M} \\
\tilde{\nabla} I^{1} \cdot v & \tilde{\nabla} I^{1} \cdot \bar{\nabla} I^{1} & \ldots & \tilde{\nabla} I^{1} \cdot \bar{\nabla} I^{M} \\
\vdots & & \ddots & \vdots \\
\tilde{\nabla} I^{M} \cdot v & \tilde{\nabla} I^{M} \cdot \bar{\nabla} I^{1} & \ldots & \tilde{\nabla} I^{M} \cdot \bar{\nabla} I^{M}
\end{array}\right) \\
& =\operatorname{det}\left(\begin{array}{c|c}
\tilde{G}^{i} \cdot v & \left(\tilde{G}^{i}\right)^{\top} \bar{D} \\
\hline \tilde{\nabla} I^{1} \cdot v & \\
\vdots & \tilde{D}^{\top} \bar{D} \\
\tilde{\nabla} I^{M} \cdot v &
\end{array}\right) \text {. }
\end{aligned}
$$


Expanding the determinant along the first column gives

$$
\begin{aligned}
& \left(\tilde{G}^{i} \wedge \tilde{\nabla} I^{1} \wedge \cdots \wedge \tilde{\nabla} I^{M}\right)_{j j_{1} \cdots j_{M}} v_{j} \bar{\nabla} I_{j_{1}}^{1} \cdots \bar{\nabla} I_{j_{M}}^{M} \\
= & \left(\tilde{G}^{i} \cdot v\right) \operatorname{det}\left(\tilde{D}^{\top} \bar{D}\right)+\sum_{j=1}^{M}(-1)^{j}\left(\tilde{\nabla} I^{j} \cdot v\right) \operatorname{det}\left(\left(\tilde{D}^{j}\right)^{\top} \bar{D}\right),
\end{aligned}
$$

where

$$
\tilde{D}^{j}=\left[\tilde{G}^{i}, \tilde{\nabla} I^{1}, \cdots, \tilde{\nabla} I^{j-1}, \tilde{\nabla} I^{j+1}, \cdots, \tilde{\nabla} I^{M}\right]
$$

Writing $\tilde{D}^{j}$ as

$$
\tilde{D^{j}}=\left[\tilde{\nabla} I^{1}, \cdots, \tilde{\nabla} I^{j-1}, \tilde{G}^{i}, \tilde{\nabla} I^{j+1}, \cdots, \tilde{\nabla} I^{M}\right]
$$

we have

$$
\tilde{S}_{j j_{1} \cdots j_{M}}^{i} v_{j} \bar{\nabla} I_{j_{1}}^{1} \cdots \bar{\nabla} I_{j_{M}}^{M}=\left[\tilde{G}^{i}-\sum_{j=1}^{M} \frac{\operatorname{det}\left(\bar{D}^{\top} \tilde{D}^{j}\right)}{\operatorname{det}\left(\bar{D}^{\top} \tilde{D}\right)} \tilde{\nabla} I^{j}\right] \cdot v .
$$

Notice that

$$
\bar{D}^{\top} \tilde{D}^{j}=\bar{D}^{\top}\left(\tilde{D}+\left(\tilde{G}^{i}-\tilde{D} e_{j}\right) e_{j}^{\top}\right)=\bar{D}^{\top} \tilde{D}+\bar{D}^{\top}\left(\tilde{G}^{i}-\tilde{D} e_{j}\right) e_{j}^{\top},
$$

where $e_{j}, j=1, \ldots, n$ are the unit vectors. By Lemma 3.2, we have

$$
\operatorname{det}\left(\bar{D}^{\top} \tilde{D}^{j}\right)=\operatorname{det}\left(\bar{D}^{\top} \tilde{D}\right)\left(1+e_{j}^{\top}\left(\bar{D}^{\top} \tilde{D}^{j}\right)^{-1} \bar{D}^{\top}\left(\tilde{G}^{i}-\tilde{D} e_{j}\right)\right),
$$

so that

$$
\begin{aligned}
\frac{\operatorname{det}\left(\bar{D}^{\top} \tilde{D^{j}}\right)}{\operatorname{det}\left(\bar{D}^{\top} \tilde{D}\right)} & =1+e_{j}^{\top}\left(\bar{D}^{\top} \tilde{D}^{j}\right)^{-1} \bar{D}^{\top}\left(\tilde{G}^{i}-\tilde{D} e_{j}\right) \\
& =1+e_{j}^{\top}\left(\bar{D}^{\top} \tilde{D}^{j}\right)^{-1} \bar{D}^{\top} \tilde{G}^{i}-e_{j}^{\top}\left(\bar{D}^{\top} \tilde{D}^{j}\right)^{-1} \bar{D}^{\top} \tilde{D} e_{j} \\
& =e_{j}^{\top}\left(\bar{D}^{\top} \tilde{D}^{j}\right)^{-1} \bar{D}^{\top} \tilde{G}^{i}
\end{aligned}
$$

Consequently,

$$
\begin{aligned}
\tilde{S}_{j j_{1} \cdots j_{M}}^{i} v_{j} \bar{\nabla} I_{j_{1}}^{1} \cdots \bar{\nabla} I_{j_{M}}^{M} & =\left[\tilde{G}^{i}-\sum_{j=1}^{M}\left(e_{j}^{\top}\left(\bar{D}^{\top} \tilde{D}^{j}\right)^{-1} \bar{D}^{\top} \tilde{G}^{i}\right) \tilde{\nabla} I^{j}\right] \cdot v \\
& =\left(\tilde{G}^{i}-\tilde{D}\left(\bar{D}^{\top} \tilde{D}^{j}\right)^{-1} \bar{D}^{\top} \tilde{G}^{i}\right) \cdot v \\
& =\left[\left(E-\tilde{D}\left(\bar{D}^{\top} \tilde{D}^{j}\right)^{-1} \bar{D}^{\top}\right) \tilde{G}^{i}\right] \cdot v \\
& =\left(P \tilde{G}^{i}\right) \cdot v .
\end{aligned}
$$

Remark 3.1. As the proof shows, the equivalence of the stochastic discrete gradient method and the stochastic projection method does not depend on the discrete gradient. Besides, the mean-square convergence of the stochastic discrete gradient method in the above theorem is equivalent to the mean-square convergence of the corresponding stochastic projection method. 


\section{Numerical Examples}

Example 4.1 (Stochastic cyclic Lotka-Volterra system). In this example, we consider the three-dimensional stochastic dynamical system in the Stratonovich sense

$$
\mathbf{d}\left(\begin{array}{l}
x(t) \\
y(t) \\
z(t)
\end{array}\right)=\left(\begin{array}{c}
x(t)(z(t)-y(t)) \\
y(t)(x(t)-z(t)) \\
z(t)(y(t)-x(t))
\end{array}\right)(\mathbf{d} t+c \circ \mathbf{d} W(t))
$$

which models three species competing in a chaotic environment [26]. Here, $c=0.5$ and initial values are $x(0)=1, y(0)=2, z(0)=1$. It is easily seen that the total derivative of functions $I^{1}(x, y, z)=x+y+z$ and $I^{2}(x, y, z)=x y z$ are equal to zero along the exact solution to (4.1). This means that $I^{1}$ and $I^{2}$ are conserved quantities of (4.1). Thus, the exact solution evolves on the manifold

$$
\mathscr{M}=\left\{(x, y, z) \mid I^{1}(x, y, z)=x+y+z=\text { const, } I^{2}(x, y, z)=x y z=\text { const }\right\},
$$

which is the intersection of a surface and a plane in $\mathbb{R}^{3}$.

Setting $G=(x(z-y), y(x-z), z(y-x))^{\top}, \nabla I^{1}=\nabla I^{1}=(1,1,1)^{\top}$ and $\nabla I^{2}=\nabla I^{2}=$ $(y z, x z, x y)^{\top}$, we arrive at a stochastic discrete gradient method for (4.1), viz.

$$
x_{j}^{\prime}=x_{j}+\frac{1}{\operatorname{det}\left(\bar{D}^{\top} \tilde{D}\right)}\left|\begin{array}{ccc}
\tilde{G}_{j} & \tilde{\nabla} I_{j}^{1} & \tilde{\nabla} I_{j}^{2} \\
\tilde{G}_{j_{1}} & \tilde{\nabla} I_{j_{1}}^{1} & \tilde{\nabla} I_{j_{1}}^{2} \\
\tilde{G}_{j_{2}} & \tilde{\nabla} I_{j_{2}}^{1} & \tilde{\nabla} I_{j_{2}}^{2}
\end{array}\right| \bar{\nabla} I_{j_{1}}^{1} \bar{\nabla} I_{j_{2}}^{2}(h+c \Delta W),
$$

where

$$
\tilde{G}=G\left(\frac{x^{\prime}+x}{2}\right), \quad \tilde{\nabla} I^{m}=\nabla I^{m}\left(\frac{x^{\prime}+x}{2}\right), \quad m=1,2,
$$

and $B=\bar{D}^{\top} \tilde{D}$ with $\bar{D}=\left[\bar{\nabla} I^{1}, \bar{\nabla} I^{2}\right]$ and $\tilde{D}=\left[\tilde{\nabla} I^{1}, \tilde{\nabla} I^{2}\right]$.

On the one hand, we apply the stochastic discrete gradient method (4.2) to (4.1) with step size $h=0.01$ and $T=100$. Fig. 1 shows the sample path of the numerical solution and the errors of the conserved quantities $I^{1}$ and $I^{2}$. Note that the conserved quantities are preserved by the stochastic discrete gradient method (4.2) and the numerical solution lies exactly on the correct manifold.

The errors of the numerical solution are defined as the mean-square sample errors at the terminal time $T=1$ over 1000 different discretised sample paths - i.e. as

$$
\sqrt{\sum_{i=1}^{1000}\left(\left|x_{T, W^{i}}^{r e f}-x_{N, W^{i}}\right|^{2}+\left|y_{T, W^{i}}^{r e f}-y_{N, W^{i}}\right|^{2}+\left|z_{T, W^{i}}^{r e f}-z_{N, W^{i}}\right|^{2}\right) / 1000 .}
$$

For each path, the stochastic discrete gradient method (4.2) is applied with five different step sizes $h=2^{-3}, h=2^{-4}, h=2^{-5}, h=2^{-6}$, and $h=2^{-7}$. Table 1 and Fig. 2 demonstrate the mean-square sample error and a log-log plot, respectively. Here, the reference solutions are obtained by mid-point method with the step size $h=2^{-12}$. Note that the stochastic discrete gradient method (4.2) converges with the mean-square order 1, consistent with the theoretical results. 



Figure 1: Numerical sample path and errors of $I^{1}$ and $I^{2}$ of the stochastic discrete gradient method (4.2) for (4.1).

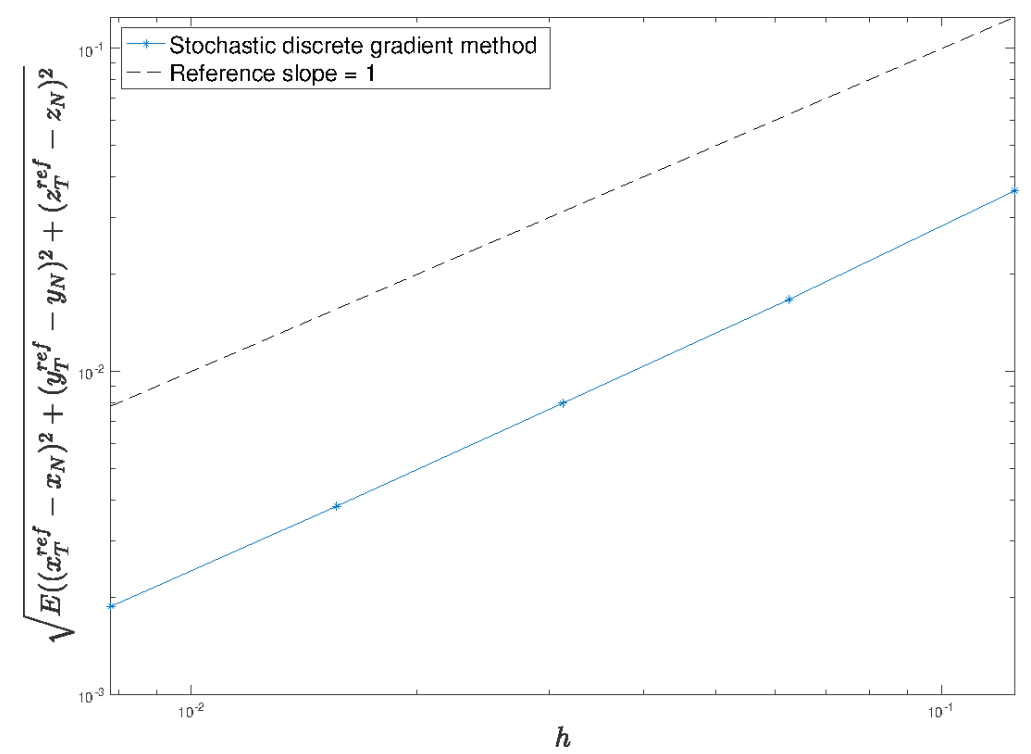

Figure 2: Convergence rate of the stochastic discrete gradient method (4.2) for (4.1).

Table 1: The mean-square error of the stochastic discrete gradient method (4.2) for solving (4.1).

\begin{tabular}{||c|c|c|c|c|c|c||}
\hline Method & $h=2^{-7}$ & $h=2^{-6}$ & $h=2^{-5}$ & $h=2^{-4}$ & $h=2^{-3}$ & Order \\
\hline Method (4.2) & $1.8729 \mathrm{e}-3$ & $3.8292 \mathrm{e}-3$ & $7.9771 \mathrm{e}-3$ & $7.9771 \mathrm{e}-2$ & $3.6242 \mathrm{e}-2$ & 1.0672 \\
\hline
\end{tabular}


Example 4.2 (Stochastic rigid body problem). This numerical example demonstrates the effectiveness of the stochastic discrete gradient method in preserving multiple conserved quantities and its equivalence to the corresponding stochastic projection method. Consider the following SDE in the Stratonovich sense driven by two Brownian motions,

$$
\begin{aligned}
\mathbf{d}\left(\begin{array}{l}
x_{1} \\
x_{2} \\
x_{3}
\end{array}\right)= & \left(\begin{array}{ccc}
0 & x_{3} / \mathscr{I}_{3} & -x_{2} / \mathscr{I}_{2} \\
-x_{3} / \mathscr{I}_{3} & 0 & x_{1} / \mathscr{I}_{1} \\
x_{2} / \mathscr{I}_{2} & -x_{1} / \mathscr{I}_{1} & 0
\end{array}\right)\left(\begin{array}{l}
x_{1} \\
x_{2} \\
x_{3}
\end{array}\right) \\
& \times\left(c_{1} \mathbf{d} t+c_{2} \circ \mathbf{d} W^{1}(t)+c_{3} \circ \mathbf{d} W^{2}(t)\right)
\end{aligned}
$$

with the initial value $\left(x_{10}, x_{20}, x_{30}\right), t \in[0, T]$ and constants $c_{1}, c_{2}, c_{3}$. The SDE (4.3) models the motion of a free rigid body affected by Brownian noises. The center of the mass located at the origin. Besides, $x_{1}, x_{2}, x_{3}$ represent the angular momenta of the body frame and $\mathscr{I}_{1}, \mathscr{I}_{2}, \mathscr{I}_{3}$ are the principal moments of inertia. Actually, the SDE (4.3) has two conserved quantities

$$
\begin{aligned}
& I^{1}\left(x_{1}, x_{2}, x_{3}\right)=x_{1}^{2}+x_{2}^{2}+x_{3}^{2}, \\
& I^{2}\left(x_{1}, x_{2}, x_{3}\right)=\frac{1}{2}\left(\frac{x_{1}^{2}}{\mathscr{I}_{1}}+\frac{x_{2}^{2}}{\mathscr{I}_{2}}+\frac{x_{3}^{2}}{\mathscr{I}_{3}}\right) .
\end{aligned}
$$

This fact can be easily established by differentiating $I^{1}$ and $I^{2}$ and substituting (4.3) into the result. Thus, we can conclude that the exact solution to (4.3) evolves on the manifold

$$
\mathscr{M}=\left\{\left(x_{1}, x_{2}, x_{3}\right) \mid x_{1}^{2}+x_{2}^{2}+x_{3}^{2}=R^{2}, \frac{1}{2}\left(\frac{x_{1}^{2}}{\mathscr{I}_{1}}+\frac{x_{2}^{2}}{\mathscr{I}_{2}}+\frac{x_{3}^{2}}{\mathscr{I}_{3}}\right)=C\right\},
$$

where $R$ and $C$ are constants. Geometrically, this means that the exact solution to (4.3) lies on the intersection of the sphere $x_{1}^{2}+x_{2}^{2}+x_{3}^{2}=R^{2}$ and ellipsoid $\frac{1}{2}\left(\frac{x_{1}^{2}}{\mathscr{I}_{1}}+\frac{x_{2}^{2}}{\mathscr{I}_{2}}+\frac{x_{3}^{2}}{\mathscr{I}_{3}}\right)=C$.

Introducing the notations

$$
\begin{aligned}
& G=\left(\left(\frac{1}{\mathscr{I}_{3}}-\frac{1}{\mathscr{I}_{2}}\right) x_{2} x_{3},\left(\frac{1}{\mathscr{I}_{1}}-\frac{1}{\mathscr{I}_{3}}\right) x_{1} x_{3},\left(\frac{1}{\mathscr{I}_{2}}-\frac{1}{\mathscr{I}_{1}}\right) x_{1} x_{2}\right)^{\top}, \\
& \nabla I^{1}=\left(2 x_{1}, 2 x_{2}, 2 x_{3}\right)^{\top}, \quad \nabla I^{2}=\left(\frac{x_{1}}{\mathscr{I}_{1}}, \frac{x_{2}}{\mathscr{I}_{2}}, \frac{x_{3}}{\mathscr{I}_{3}}\right)^{\top},
\end{aligned}
$$

we write the stochastic discrete gradient method for (4.3) as

$$
x_{j}^{\prime}=x_{j}+\frac{1}{\operatorname{det}\left(\bar{D}^{\top} \tilde{D}\right)}\left|\begin{array}{ccc}
\tilde{G}_{j} & \tilde{\nabla} I_{j}^{1} & \tilde{\nabla} I_{j}^{2} \\
\tilde{G}_{j_{1}} & \tilde{\nabla} I_{j_{1}}^{1} & \tilde{\nabla} I_{j_{1}}^{2} \\
\tilde{G}_{j_{2}} & \tilde{\nabla} I_{j_{2}}^{1} & \tilde{\nabla} I_{j_{2}}^{2}
\end{array}\right| \bar{\nabla} I_{j_{1}}^{1} \bar{\nabla} I_{j_{2}}^{2}\left(c_{1} h+c_{2} \Delta W^{1}+c_{3} \Delta W^{2}\right)
$$

where

$$
\tilde{G}=G\left(\frac{x^{\prime}+x}{2}\right), \quad \tilde{\nabla} I^{m}=\nabla I^{m}\left(\frac{x^{\prime}+x}{2}\right), \quad m=1,2,
$$

and $B=\bar{D}^{\top} \tilde{D}$ with $\bar{D}=\left[\bar{\nabla} I^{1}, \bar{\nabla} I^{2}\right], \tilde{D}=\left[\tilde{\nabla} I^{1}, \tilde{\nabla} I^{2}\right]$. 

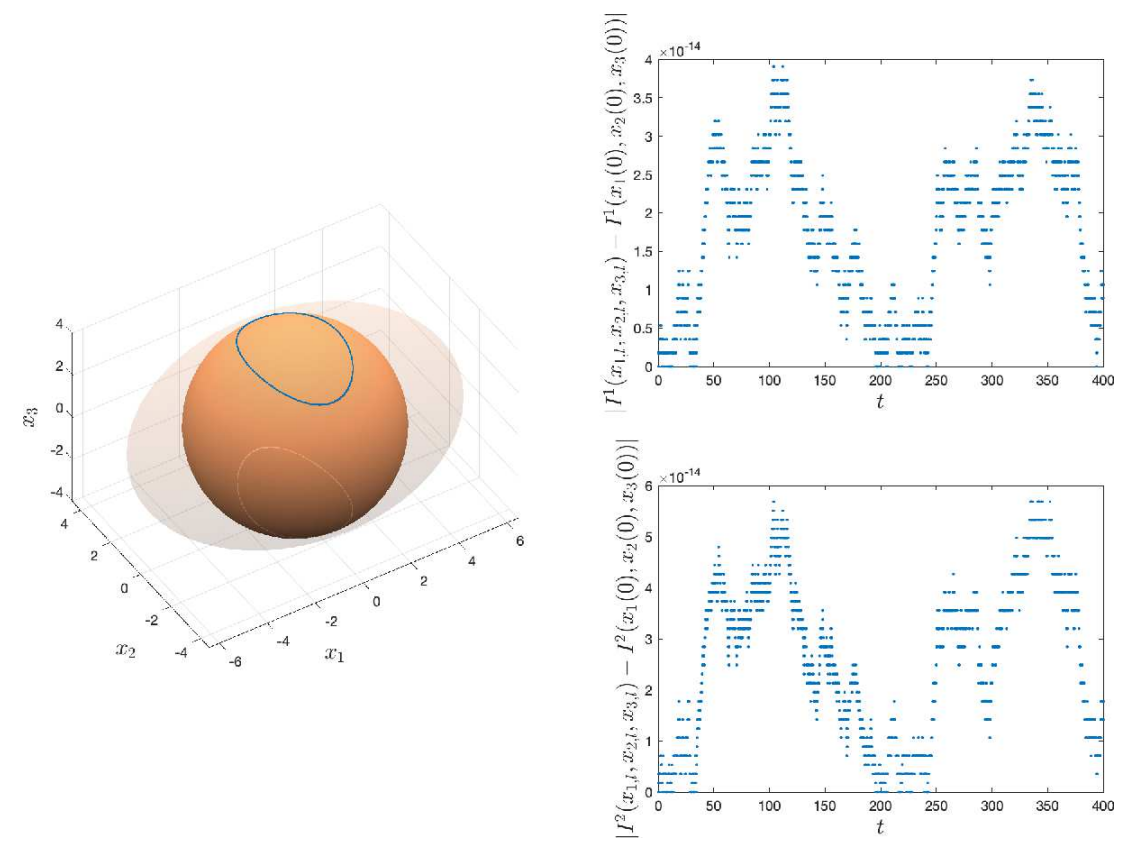

Figure 3: Numerical sample path and errors in $I^{1}$ and $I^{2}$ of the stochastic discrete gradient method (4.4) for (4.3).

Choosing the parameters $c_{1}=1, c_{2}=0.1, c_{3}=0.1, R=4, \mathscr{I}_{1}=2, \mathscr{I}_{2}=1, \mathscr{I}_{3}=2 / 3$, $T=400$ and the initial values $x_{10}=R \cos (1.1), x_{20}=0, x_{30}=R \sin (1.1)$, we apply the stochastic discrete gradient method (4.4) and the corresponding stochastic projection method (3.7) to the problem (4.3) with the step size $h=0.1$. Figs. 3 and 4 show the sample path and the errors of the conserved quantities $I^{1}$ and $I^{2}$ for the stochastic discrete gradient method (4.4) and the corresponding stochastic projection method (3.7). The CPU time of the methods (4.4) and (3.7) are 5.85575 and 7.10106 seconds, respectively. Observe that the conserved quantities are preserved well by the methods (3.7) and (4.4), and in both cases the numerical solutions lie on the correct manifold - i.e. on the intersection of the sphere and ellipsoid. Besides, Fig. 5 displays the difference between the numerical solutions obtained by the stochastic discrete gradient method (4.4) and the corresponding stochastic projection method (3.7). Fig. 5 shows that the methods are equivalent.

\section{Conclusions}

We extend the deterministic discrete gradient method to the stochastic counterpart for solving SDEs with multiple conserved quantities. The SDEs with multiple conserved quantities in the Stratonovich sense are written in the skew-gradient form, which is used in the construction of the stochastic discrete gradient method. It is shown that the stochastic discrete gradient method has the mean-square convergence order 1 and preserves all conserved quantities of the original SDEs. Besides, for a given skew-gradient form, the cor- 

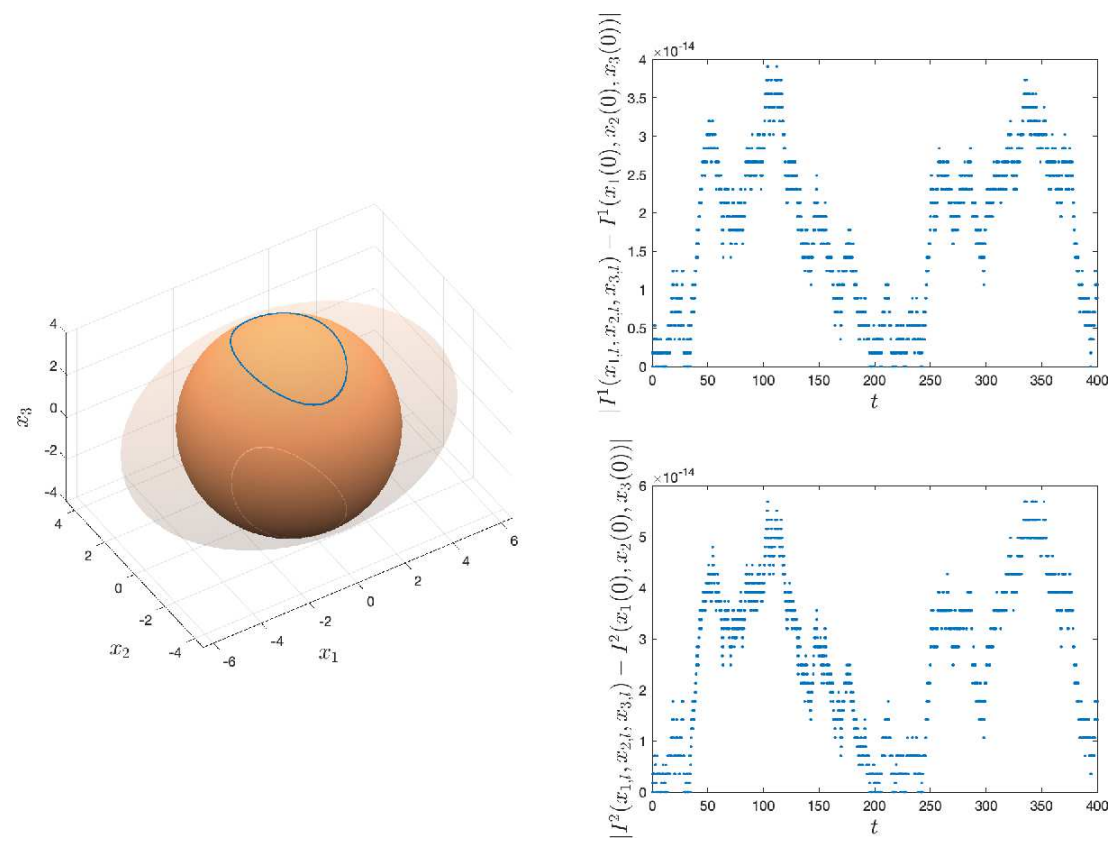

Figure 4: Numerical sample path and errors in $I^{1}$ and $I^{2}$ of the stochastic projection method (3.7) for solving (4.3).
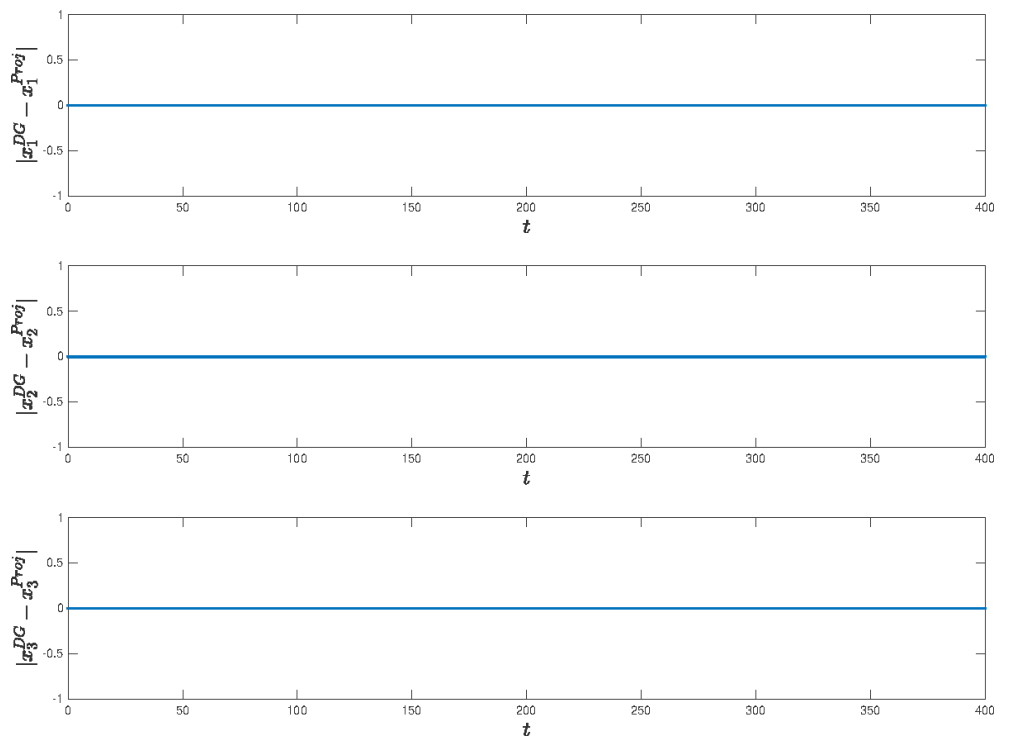

Figure 5: The difference between the stochastic discrete gradient method (4.4) and stochastic projection method (3.7) for solving (4.3).

responding stochastic discrete gradient method is equivalent to the stochastic projection method. Numerical examples confirm the theoretical results and show the effectiveness of the method. 


\section{Acknowledgments}

This work is supported by the Natural Science Foundation of Shandong Province of China under Grant ZR2020MA050 and by the National Natural Science Foundation of China under Grant 11901355.

\section{References}

[1] K. Burrage and P. Burrage, High strong order methods for non-commutative stochastic ordinary differential equation systems and the Magnus formula, Phys. D 133, 34-48 (1999).

[2] K. Burrage and P.M. Burrage, Order conditions of stochastic Runge-Kutta methods by B-series, SIAM J. Numer. Anal. 38, 1626-1646 (2000).

[3] W. Cao, Z. Zhang and G.E. Karniadakis, Numerical methods for stochastic delay differential equations via the Wong-Zakai approximation, SIAM J. Sci. Comput. 37, A295-A31837 (2015).

[4] C. Chen, J. Hong and D. Jin, Modified averaged vector field methods preserving multiple invariants for conservative stochastic differential equations, BIT 60, 1-41 (2020).

[5] S.H. Christiansen, H.Z. Munthe-Kaas and B. Owren, Topics in structure-preserving discretization, Acta Numer. 20, 1-119 (2011).

[6] D. Cohen and G. Dujardin, Energy-preserving integrators for stochastic Poisson systems, Commun. Math. Sci. 12, 1523-1539 (2014).

[7] J.A. Freund and T. Pöschel, Stochastic Processes in Physics, Chemistry, and Biology, Springer (2000).

[8] E. Hairer, C. Lubich and G. Wanner, Geometric Numerical Integration: Structure-Preserving Algorithms for Ordinary Differential Equations, Springer (2006).

[9] J. Hong, J. Ruan, L. Sun and L. Wang, Structure-preserving numerical methods for stochastic Poisson systems, ArXiv: 2006.03880 (2020).

[10] J. Hong, S. Zhai and J. Zhang, Discrete gradient approach to stochastic differential equations with a conserved quantity, SIAM J. Numer. Anal. 49, 2017-2038 (2011).

[11] L. Hu and S. Gan, Numerical analysis of the balanced implicit methods for stochastic pantograph equations with jumps, Appl. Math. Comput. 223, 281-297 (2013).

[12] D. Li and M. Liu, Invariant measure of a stochastic food-limited population model with regime switching, Math. Comput. Simulation 2020, 16-26 (2020).

[13] M. Li and C. Huang, Projected Euler-Maruyama method for stochastic delay differential equations under a global monotonicity condition, Appl. Math. Comput. 366, 124733 (2020).

[14] X. Li, Q. Ma and X. Ding, High-order energy-preserving methods for stochastic Poisson systems, East Asian J. Appl. Math. 9, 465-484 (2019).

[15] X. Li, C. Zhang, Q. Ma and X. Ding, Discrete gradient methods and linear projection methods for preserving a conserved quantity of stochastic differential equations, Int. J. Comput. Math. 95, 2511-2524 (2018).

[16] X. Li, C. Zhang, Q. Ma and X. Ding, Arbitrary high-order equip methods for stochastic canonical Hamiltonian systems, Taiwanese J. Math. 23, 703-725 (2019).

[17] M. Liu and M. Deng, Analysis of a stochastic hybrid population model with allee effect, Appl. Math. Comput. 364, 124582 (2020).

[18] M. Liu and P.S. Mandal, Dynamical behavior of a one-prey two-predator model with random perturbations, Commun. Nonlinear Sci. Numer. Simul. 28, 123-137 (2015).

[19] S.J.A. Malham and A. Wiese, Stochastic Lie group integrators, SIAM J. Sci. Comput. 30, 597617 (2008). 
[20] X. Mao, Stochastic Differential Equations and Applications, Horwood Publishing (2007).

[21] R.I. McLachlan, G.R.W. Quispel and N. Robidoux, Geometric integration using discrete gradients, Philos. Trans. Roy. Soc. A 357, 1021-1045 (1999).

[22] C.D. Meyer, Matrix Analysis and Applied Linear Algebra, SIAM (2000).

[23] G.N. Milstein, Numerical Integration of Stochastic Differential Equations, Kluwer Academic Publishers (1995).

[24] G.N. Milstein, Y.M. Repin and M.V. Tretyakov, Numerical methods for stochastic systems preserving symplectic structure, SIAM J. Numer. Anal. 35, 1583-1604 (2002).

[25] G.N. Milstein and M.V. Tretyakov, Stochastic Numerics for Mathematical Physics, Springer (2013).

[26] T. Misawa, Conserved quantities and symmetries related to stochastic dynamical systems, Ann. Inst. Statist. Math. 103, 239-248 (2002).

[27] H.Z. Munthe-Kaas, A. Stern and O. Verdier, Invariant connections, Lie algebra actions, and foundations of numerical integration on manifolds, SIAM J. Appl. Algebra Geom. 4, 49-68 (2020).

[28] Y. Sun and W. Zhao, New second-order schemes for forward backward stochastic differential equations, East Asian J. Appl. Math. 8, 399-421 (2018).

[29] X. Wang, An efficient explicit full-discrete scheme for strong approximation of stochastic AllenCahn equation, Stochastic Process. Appl. 130, 6271-6299 (2020).

[30] X. Wang, J. Wu and B. Dong, Mean-square convergence rates of stochastic theta methods for SDEs under a coupled monotonicity condition, BIT 2, 1-32 (2020).

[31] Z. Wang, C. Wang, Q. Ma and X. Ding, Numerical simulations for stochastic differential equations on manifolds by stochastic symmetric projection method, Phys. A 541, 123305 (2020).

[32] A. Zanna, Explicit volume-preserving splitting methods for divergence-free ODEs by tensorproduct basis decompositions, IMA J. Numer. Anal. 35, 89-106 (2015).

[33] W. Zhao and M. Gunzburger, Auxiliary equations approach for the stochastic unsteady NavierStokes equations with additive random noise, Numer. Math. Theory Methods Appl. 13, 1-26 (2020).

[34] W. Zhou, L. Zhang, J. Hong and S. Song, Projection methods for stochastic differential equations with conserved quantities, BIT 56, 1497-1518 (2016). 